\title{
Human mitotic chromosomes consist predominantly of irregularly folded nucleosome fibres without a 30-nm chromatin structure
}

\author{
Yoshinori Nishino ${ }^{1,2,9}$, Mikhail Eltsov ${ }^{3,9}$, \\ Yasumasa Joti ${ }^{4,9}$, Kazuki Ito ${ }^{1,9}$, \\ Hideaki Takata ${ }^{5}$, Yukio Takahashi ${ }^{1,6}$, \\ Saera Hihara ${ }^{5,7}$, Achilleas S Frangakis, \\ Naoko Imamoto ${ }^{8}$, Tetsuya Ishikawa ${ }^{1,4}$ \\ and Kazuhiro Maeshima ${ }^{1,5,7,8, *}$
}

\begin{abstract}
${ }^{1}$ RIKEN SPring-8 Center, Hyogo, Japan, ${ }^{2}$ Research Institute for Electronic Science, Hokkaido University, Sapporo, Japan, ${ }^{3}$ European Molecular Biology Laboratory, Heidelberg, Germany, ${ }^{4}$ RIKEN, XFEL Project Head Office, Hyogo, Japan, ${ }^{5}$ Biological Macromolecules Laboratory, Structural Biology Center, National Institute of Genetics, Shizuoka, Japan ${ }^{6}$ Graduate School of Engineering, Osaka University, Osaka, Japan, ${ }^{7}$ Department of Genetics, School of Life Science, Graduate University for Advanced Studies (Sokendai), Shizuoka, Japan and ${ }^{8}$ Cellular Dynamics Laboratory, RIKEN Advanced Science Institute, Saitama, Japan
\end{abstract}

How a long strand of genomic DNA is compacted into a mitotic chromosome remains one of the basic questions in biology. The nucleosome fibre, in which DNA is wrapped around core histones, has long been assumed to be folded into a 30-nm chromatin fibre and further hierarchical regular structures to form mitotic chromosomes, although the actual existence of these regular structures is controversial. Here, we show that human mitotic HeLa chromosomes are mainly composed of irregularly folded nucleosome fibres rather than $30-\mathrm{nm}$ chromatin fibres. Our comprehensive and quantitative study using cryoelectron microscopy and synchrotron $\mathrm{X}$-ray scattering resolved the long-standing contradictions regarding the existence of 30-nm chromatin structures and detected no regular structure $>11 \mathrm{~nm}$. Our finding suggests that the mitotic chromosome consists of irregularly arranged nucleosome fibres, with a fractal nature, which permits a more dynamic and flexible genome organization than would be allowed by static regular structures.

The EMBO Journal (2012) 31, 1644-1653. doi:10.1038/

emboj.2012.35; Published online 17 February 2012

Subject Categories: chromatin \& transcription

Keywords: chromosome structure; cryo-EM; fractal structure; X-ray sacattering; 30-nm chromatin fibre

\section{Introduction}

During eukaryotic cell division, mitotic chromosomes are formed to ensure the faithful transmission of replicated genomic DNA into two daughter cells (Alberts et al, 2007).

\footnotetext{
*Corresponding author. Biological Macromolecules Laboratory, Structural Biology Center, National Institute of Genetics, Mishima, Shizuoka 411-8540, Japan. Tel.: +81 55981 6864;

Fax: + 8155981 6865; E-mail: kmaeshim@lab.nig.ac.jp

${ }^{9}$ These four authors contributed equally to this work
}

Received: 31 October 2011; accepted: 26 January 2012; published online: 17 February 2012
A long strand of DNA is wrapped around core histones to form a nucleosome structure like 'beads on a string' (Kornberg and Lorch, 1999). It has long been assumed that this nucleosome fibre is folded into $30-\mathrm{nm}$ chromatin fibres (Alberts et al, 2007) and that condensins are involved in further regular chromatin folding (Swedlow and Hirano, 2003; Kireeva et al, 2004), although the folding processes remain unclear (Hirano, 2006; Tremethick, 2007; Maeshima and Eltsov, 2008; Ohta et al, 2010).

Several models of chromatin fibres have been proposed, the diameters of which are around $30 \mathrm{~nm}$ (Finch and Klug, 1976; Schalch et al, 2005; Robinson et al, 2006). Extensive data based on biochemistry, conventional electron microscopy (EM) and small-angle X-ray scattering (SAXS) support various 30-nm chromatin fibre structures (Finch and Klug, 1976; Langmore and Paulson, 1983; Woodcock et al, 1984; Widom and Klug, 1985; Bordas et al, 1986; Gilbert et al, 2004; Schalch et al, 2005; Robinson et al, 2006). However, cryo-EM studies have demonstrated 30-nm chromatin fibre structures in only a few highly specialized cell types (Woodcock, 1994), and cryo-EM data have argued strongly against the existence of $30-\mathrm{nm}$ chromatin structures in mitotic chromosomes in situ (McDowall et al, 1986; Eltsov et al, 2008; Maeshima and Eltsov, 2008).

To resolve these long-standing discrepancies, we performed a comprehensive and quantitative investigation of the mitotic chromosome structure using cryo-EM, SAXS and ultra-SAXS (USAXS). SAXS analysis detects periodic structures in biological materials in solution. Cryo-EM allows one to observe biological samples in a frozen hydrated state, although conventional EM can observe only fixed and dehydrated samples, which can produce various potential artefacts (Dubochet and Sartori Blanc, 2001; Maeshima et al, 2010b). Our data demonstrate that the $30-\mathrm{nm}$ structures observed in SAXS studies are due to contamination by regularly spaced ribosome aggregates and do not originate from the chromosomes themselves. Also, no regular periodic structure $>11 \mathrm{~nm}$ was detected within a range extending up to the diameter of whole chromosomes. We suggest that the mitotic chromosome consists of irregularly arranged nucleosome fibres, with a fractal nature.

\section{Results}

\section{$X$-ray scattering profile of mitotic HeLa chromosomes}

To explore possible periodic structures including 30-nm fibres in mitotic chromosomes, we performed SAXS measurements of mitotic HeLa chromosomes using the synchrotron X-ray source at SPring-8. As previous reports suggested that chicken erythrocyte nuclei, which are almost completely transcriptionally silenced, contain 30-nm chromatin fibres (Langmore and Schutt, 1980; Woodcock, 1994), these were used as positive controls in the present study. 

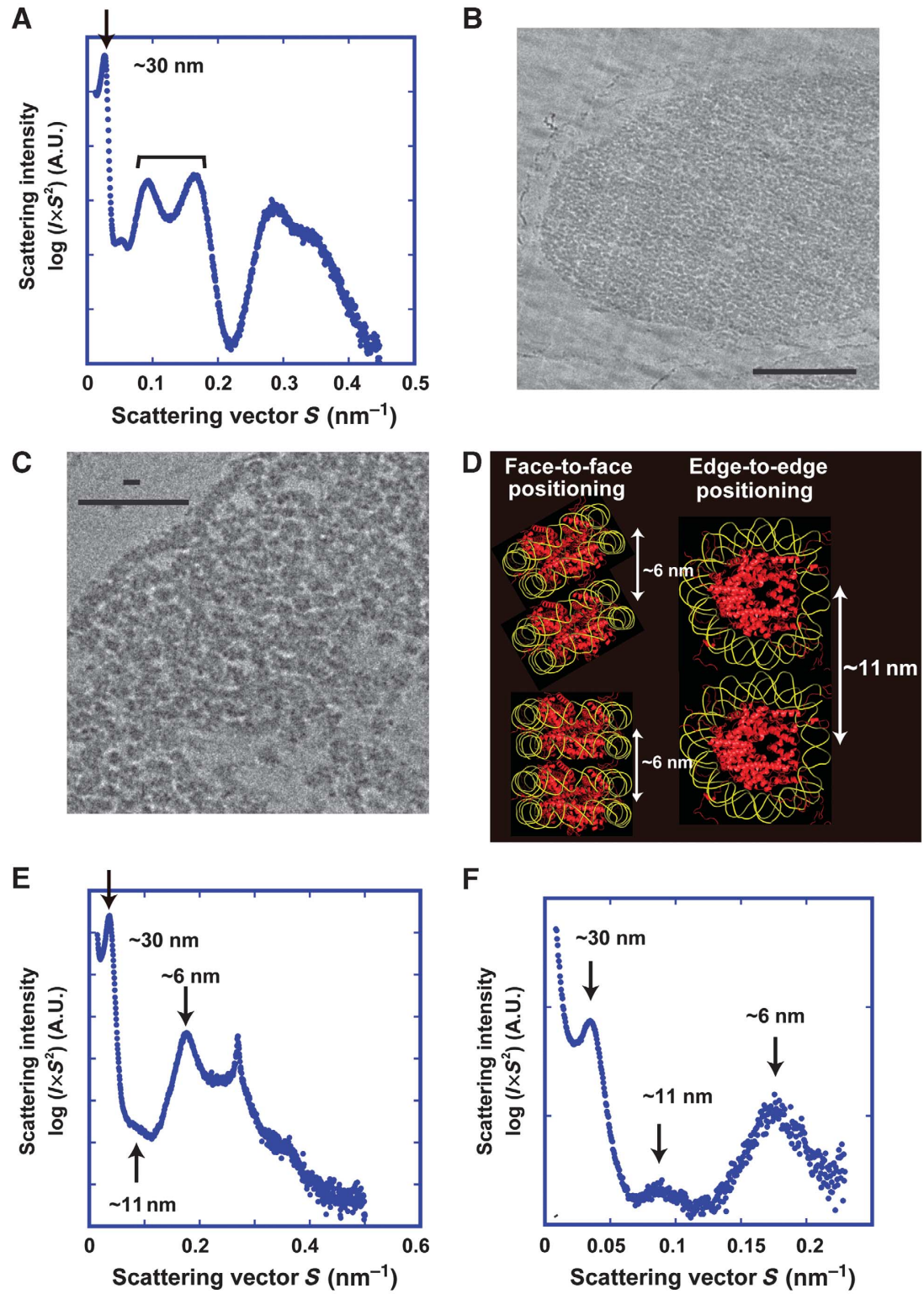

Figure 1 SAXS profile of mitotic HeLa chromosomes. (A) A typical SAXS pattern of the chicken erythrocyte nuclei using the BL45XU beamline at SPring-8. In the plot of $\log \left(I \times S^{2}\right)$ versus $S, I$ is the measured average intensity and $S$ is the size of the scattering vector, the inverse of the structure or spacing size (for details, see Materials and methods). Note that as size variations may exist in the structures, peaks in the measurements may also exhibit some variation. The chicken erythrocyte nuclei produced a sharp 30-nm peak (arrow). In addition, two peaks of $\sim 11$ and $\sim 6 \mathrm{~nm}$ were prominent (brackets). (B) Dot-like 30-nm structures in the chicken erythrocyte nucleus were observed by cryo-EM (see also Supplementary Figure S1). Bar indicates $5 \mu \mathrm{m}$. (C) High-resolution cryo-EM image of a chicken erythrocyte nucleus. Long and short bars indicate 200 and $30 \mathrm{~nm}$, respectively. (D) Two types of nucleosome positioning: face-to-face, with 6-nm spacing, and edge-to-edge, with $\sim 11$-nm spacing. Note that in helical fibres such as the 30-nm chromatin fibre, edge-to-edge spacing likely corresponds to helical pitch (Figure 6A). The nucleosome model was taken from Davey et al (2002) (yellow, DNA; red, core histones). (E, F) Typical SAXS patterns of mitotic HeLa chromosomes. In (E), a SAXS scattering pattern covering larger angles is shown to reveal smaller structures. Three peaks at $\sim 6$, $\sim 11$ and $\sim 30 \mathrm{~nm}$ were detected (arrows) (see also Supplementary Figure S2A and B).

Table I Comparison of the scattering profile between erythrocyte nuclei (Figure 1A), modelled 30-nm chromatin fibres (Figure 5A), mitotic HeLa chromosomes with ribosomes (Figure 3C) and mitotic HeLa chromosomes without ribosomes (Figure 3C)

$$
\sim 30 \text {-nm Peak } \quad \sim 11-n m \text { Peak }
$$
$\sim 6-\mathrm{nm}$ Peak

Erythrocyte nuclei (30-nm chromatin fibres)

Single 30-nm chromatin fibre simulation (two-start)

Mitotic HeLa chromosomes (with ribosomes)

Mitotic HeLa chromosomes (without ribosomes)

$\begin{array}{ccc}+++ & +++ & +++ \\ \mathrm{ND} & ++++ & +++ \\ +++ & + & +++ \\ - & + & +++\end{array}$

Relative peak intensity is represented as ' +'. ND, not determined. 
A
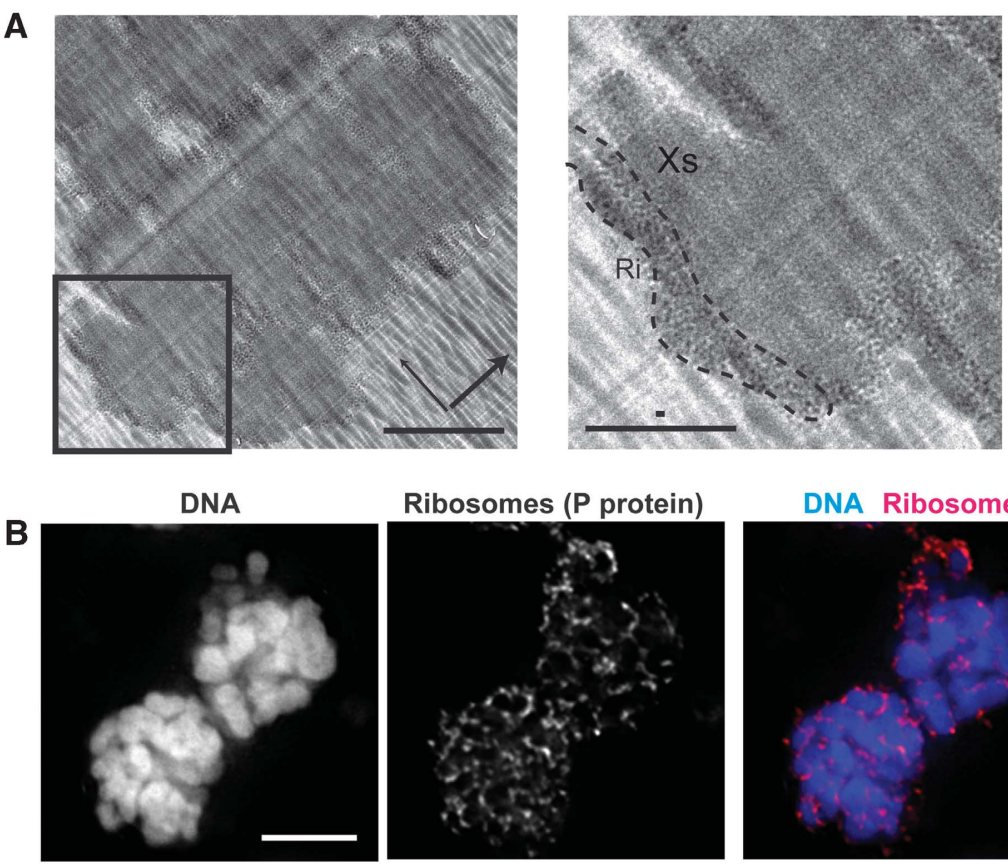

Ribosomes (P protein)
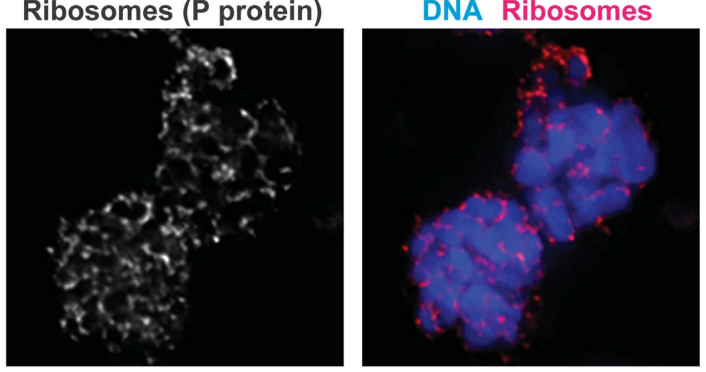

C

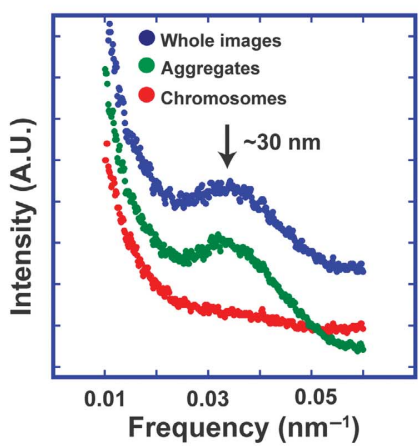

D

Before washing

After washing

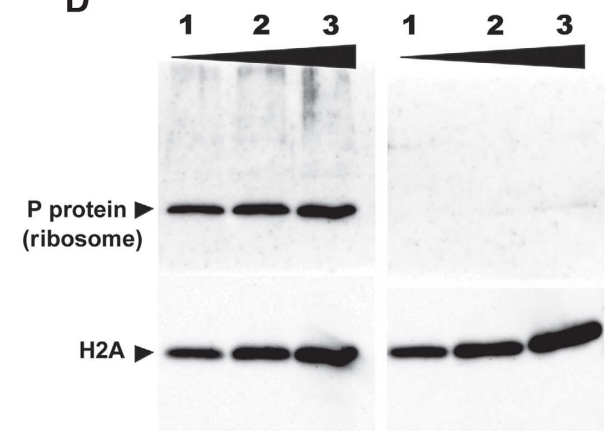

Figure 2 Ribosome aggregates around chromosomes. (A) Cryo-EM image of the chromosome clusters (cross-sections). Many black dots were observed on the chromosome surface. Note that knife marks (thick arrow) and compression marks (thin arrow) were observed. Bars indicate $1 \mu \mathrm{m}$. An enlarged image of the region outlined with a box is depicted on the right. A black dot region is surrounded by a broken line (Ri). A chromosome part is marked as 'Xs'. Long and short bars indicate $0.5 \mu \mathrm{m}$ and $30 \mathrm{~nm}$, respectively. (B) Immunostaining with anti-P antibody against a ribosomal component suggested that the peripheral black dots were ribosome aggregates (Uchiumi et al, 1990). DNA (DAPI), a ribosome and merged images of two chromosome clusters are shown. Bar indicates $10 \mu \mathrm{m}$ (see also (D) and Supplementary Figure S3). (C) Power spectrum (Fourier transform) analyses of the aggregated regions, the chromosome regions and whole cryo-EM images. A 30-nm peak (arrow) was seen in the aggregated region (green dots) and whole images (blue dots). (D) Ribosome removal was verified by western blotting (Uchiumi et al, 1990).

In SAXS measurements, membranous structures, including small vesicles, generate scattering maxima at $30-40 \mathrm{~nm}$ that add a great deal of 'noise' to the signal; therefore, mitotic HeLa chromosomes and chicken erythrocyte nuclei were isolated in physiological salt buffer from mitotic HeLa cells and chicken erythrocytes, respectively (Langmore and Schutt, 1980; Langmore and Paulson, 1983; Maeshima et al, 2005). We did not carry out any chemical fixation or alcohol dehydration, which are common in conventional EM sample processing, to avoid possible artefacts caused by such treatments (Dubochet and Sartori Blanc, 2001; Maeshima et al, $2010 \mathrm{~b})$. The samples were placed in a quartz glass capillary and exposed to an X-ray beam for $1 \mathrm{~s}$, during which the scattering patterns were recorded. Several sequential exposures to the X-ray beam did not change the profile of the scattering pattern (Maeshima, unpublished data), confirming that no significant radiation damage to the chromatin structure had occurred.
SAXS analysis of the erythrocyte nuclei revealed a sharp 30-nm peak and two prominent peaks at $\sim 11$ and $\sim 6 \mathrm{~nm}$ (Figure 1A; Table I). We also detected apparent 30-nm structures in the chicken erythrocyte nuclei by cryo-EM (Figure 1B and C), and a further power spectrum analysis of the chromatin regions revealed periodic structures (Supplementary Figure S1). These results were consistent with those of previous reports (Langmore and Schutt, 1980; Woodcock, 1994). Therefore, we concluded that we should see 30-nm structures by SAXS and cryo-EM if they indeed exist.

We next focused on the mitotic HeLa chromosomes. Almost 30 years ago, Langmore and Paulson (1983) reported SAXS peaks at $\sim 30, \sim 11$ and $\sim 6 \mathrm{~nm}$ in the mitotic chromosomes. They concluded that the 6 - and $11-\mathrm{nm}$ peaks were derived from the positioning of nucleosomes on their flat faces and from edge-to-edge positioning, respectively (Figure 1D) (Langmore and Paulson, 1983). These authors also suggested that the 30 -nm peak represented the side-by-side 
A

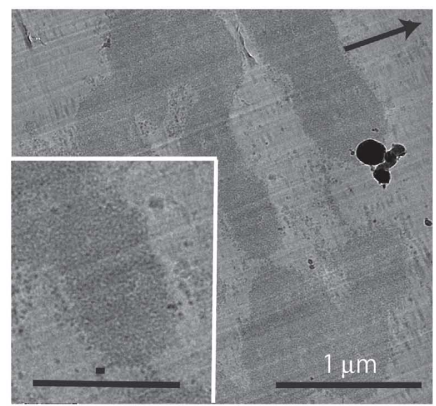

C
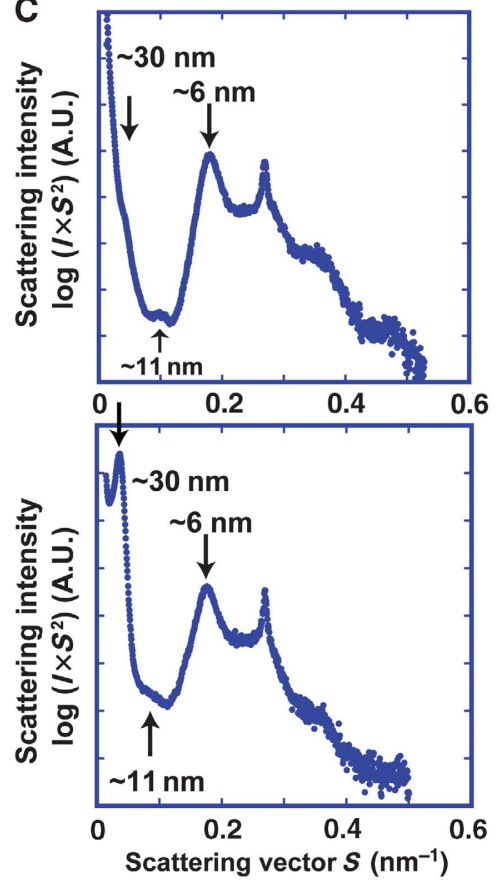

B
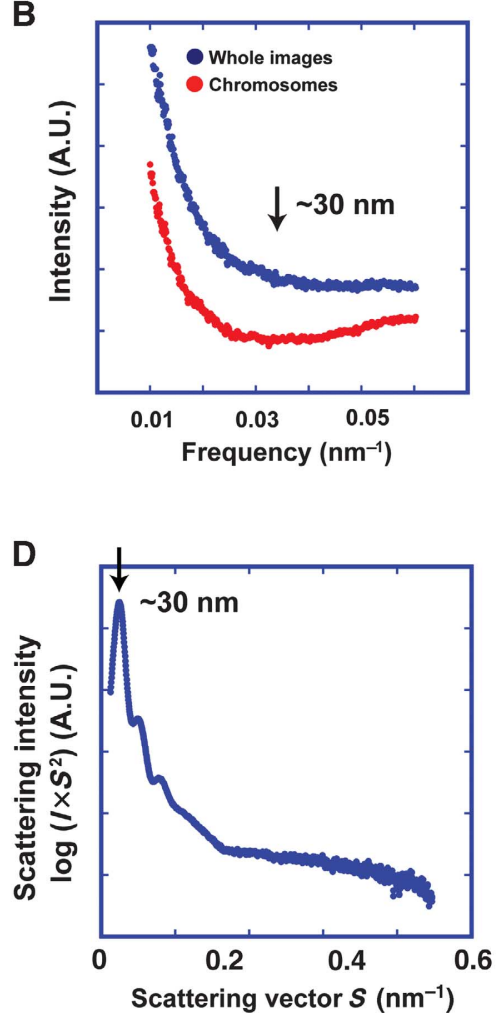
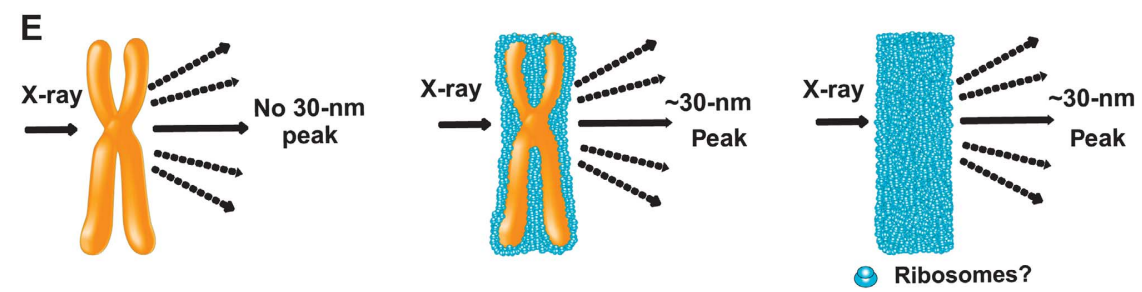

Figure 3 The 30-nm peak on the SAXS profile was derived from ribosome aggregates. (A) Removal of ribosome aggregates. Isolated chromosomes were washed in buffer A (polyamine + EDTA) (Lewis and Laemmli, 1982; Paulson and Langmore, 1983; Maeshima and Laemmli, 2003) and then returned to IB containing $5 \mathrm{mM} \mathrm{Mg}^{2+}$ (Paulson and Langmore, 1983). Cryo-EM showed that this treatment removed most of the aggregates. Note that knife marks (arrow) were observed. Bar indicates $1 \mu \mathrm{m}$. The inset shows an enlarged image. Long and short bars indicate $0.5 \mu \mathrm{m}$ and $30 \mathrm{~nm}$, respectively. (B) Power spectrum (Fourier transform) analysis of cryo-EM images after removal of ribosome aggregates. Neither whole images (blue dots) nor chromosome regions (red dots) showed a peak at $\sim 30 \mathrm{~nm}$ (arrow). (C) In SAXS analysis, only the 30-nm peak disappeared after removal of ribosome aggregates (upper), whereas the other peaks remained. For comparison, Figure 1E is reproduced (lower). (D) A ribosomal fraction from Xenopus egg extracts (kindly provided by Dr T Hirano, RIKEN) produced a 30-nm peak (arrow) on SAXS. (E) Model explaining the results: the 30-nm peak in SAXS comes from regularly spaced aggregates of ribosomes, and not from the chromosomes themselves.

packing of 30-nm chromatin fibres, which has long been regarded as strong evidence for the existence of these fibres in chromosomes. Consistent with their data, we detected peaks at $\sim 30, \sim 11$ and $\sim 6 \mathrm{~nm}$ as shown in Figure $1 \mathrm{E}$ and $\mathrm{F}$ that show the peaks in the larger structures more clearly. In contrast to the erythrocyte nuclei, the $\sim 6$-nm peak was prominent, whereas the $\sim 11-\mathrm{nm}$ peak was rather faint (see also Supplementary Figure S2A and B and Table I).

\section{The 30-nm SAXS peak originates from ribosome aggregations}

Although we successfully reproduced the earlier SAXS findings (Figure 1E and F; Supplementary Figure S2A and B), in a previous cryo-EM study, we found no evidence of $30-\mathrm{nm}$ structures in mitotic chromosomes (Eltsov et al, 2008; Maeshima and Eltsov, 2008), indicating a striking inconsistency between the two techniques. To identify the source of 

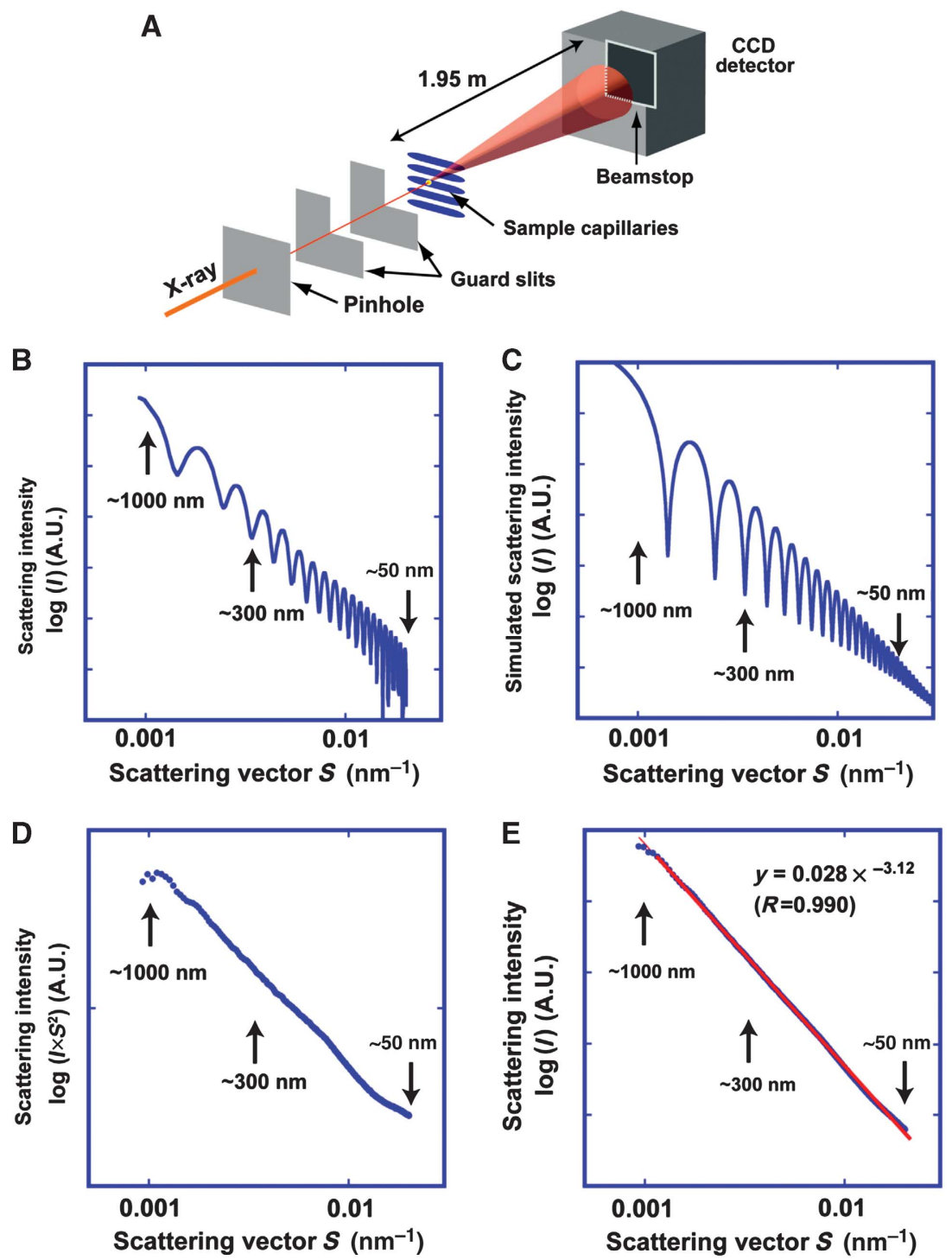

Figure 4 Mitotic chromosomes lack notable higher-order structures. (A) Experimental setup of USAXS using the BL29XUL beamline at SPring-8 (for details, see Materials and methods). This system accomplished efficient USAXS measurements using a compact apparatus. (B) USAXS study of latex particles with diameters of $\sim 1000 \mathrm{~nm}$ showed clear fringes over a wide range $(\sim 50-1000 \mathrm{~nm})$. The profile is plotted as $\log (I)$ versus $\log (S)$. (C) A computer simulation of latex particles with diameters of $\sim 1000 \mathrm{~nm}$ produced a pattern highly similar to that measured. (D) By USAXS, no notable structures around $100-150$ or $200-250 \mathrm{~nm}$ were detected (see also Supplementary Figure S5A). (E) The scattering intensity obeyed the power law with respect to structure size and spacing. To determine the nature of the structure at sizes over $11 \mathrm{~nm}$, the logarithm of the scattering intensity $(I)$ and size of the scattering vector $(S)$ were taken from the data in (D) (Schmidt, 1989). A plot of log $(I)$ versus $\log (S)$ on a straight line (red line) covered a wide range, extending over nearly four orders of magnitude. Least-squares fitting showed that $I$ is proportional to $S$ to the power of $-3.12(R=0.990)$ (Schmidt, 1989), suggesting that chromosomes do not possess notable regular structures over a very wide scale and exhibit a fractal nature of genome organization (see also Supplementary Figure S5B).

the 30-nm SAXS peak, we examined isolated chromosome samples by cryo-EM (Figure $2 \mathrm{~A}$ ) using the chromosome isolation procedure of Langmore and Paulson (1983). Unexpectedly, we observed extensive aggregates of electrondense granules attached to the chromosome surface (chromosome region:aggregate region $=1: 0.35$ ) (Figure $2 \mathrm{~A}$ ). Based on their size, these granules were very likely to be ribosomes; to further test this suggestion, we performed immunostaining (Figure 2B; Supplementary Figure S3) and western blotting (Figure 2D) (Uchiumi et al, 1990).

The ribosome particles in the aggregates seemed to be regularly spaced (Figure 2A). To detect the periodicity on the cryo-EM images, we performed power spectra (Fourier transform) analyses. When the chromosome regions were selectively analysed, we did not detect a 30-nm peak, consistent with our previous finding (Eltsov et al, 2008). However, the ribosome aggregates showed a prominent $\sim 30$-nm peak (Figure 2C). The 30-nm signal from the ribosome region was so strong that the whole image still produced a notable $30-\mathrm{nm}$ peak (Figure 2C). This result suggested that the $30-\mathrm{nm}$ peak in SAXS resulted from ribosome aggregates (Figure 2C).

To further test whether the ribosome aggregates were responsible for the 30-nm SAXS peak, we removed them by washing the chromosomes with an isotonic buffer containing polyamine and EDTA (buffer A) (Lewis and Laemmli, 1982; Paulson and Langmore, 1983; Maeshima and Laemmli, 2003). This buffer maintains the size and shape of chromosomes 
A

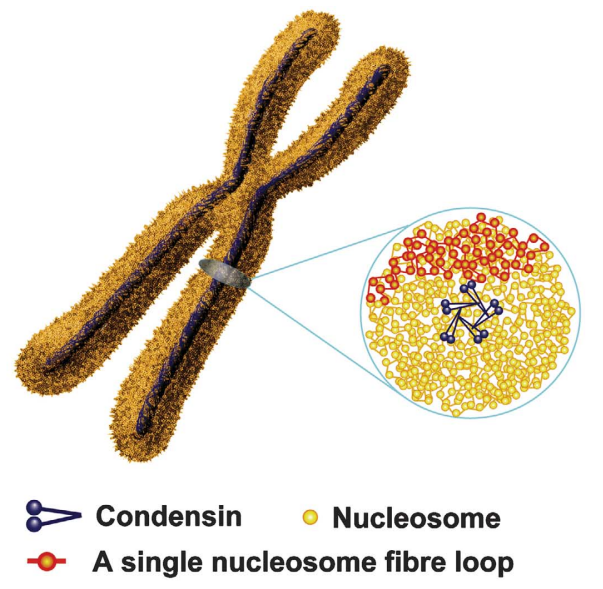

B

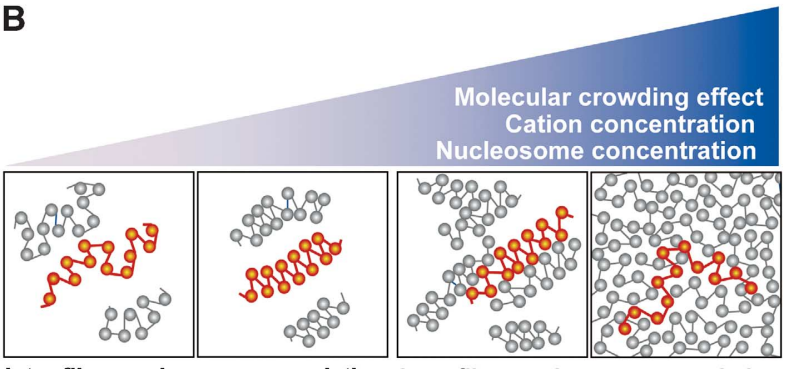

Intra-fibre nucleosome association Inter-fibre nucleosome association

Figure 5 Chromosome structure model. (A) Chromosomes consist essentially of irregularly folded nucleosome (beads on a string) fibres. Condensins (blue) hold the nucleosome fibres (red) globally around the chromosome centre. Locally, the nucleosome fibre is folded in an irregular or disordered manner, forming loop structures that are collapsed towards the chromosome centre (blue). The collapsed fibre (red) forms a domain that could be compatible with the large module observed by the Belmont group (Strukov et al, 2003). (B) Dynamic melted nucleosome. Under dilute conditions with low nucleosome fibre and cation concentrations, nucleosome fibres can form 30-nm chromatin fibres via intrafibre nucleosome associations. An increase in fibre concentration, as a consequence of an increase in cation concentration and/or a molecular crowding effect, results in inter-fibre nucleosomal contacts that interfere with intra-fibre nucleosomal associations. Nucleosomes of adjacent fibres interdigitate, leading to a polymer melt-like structure. Note that in these illustrations, we show a highly simplified two-dimensional nucleosome model, which does not show the details of chromatin fractal organization.

(Supplementary Figure S4). The preparation was then resuspended in the original isolation buffer (IB) (Langmore and Paulson, 1983). This treatment removed most of the ribosome aggregates from the chromosome surfaces (Figure 3A), consistent with our biochemical data (Figure 2D). As expected, the $30-\mathrm{nm}$ peak in the power spectra and SAXS pattern disappeared almost completely (Figure $3 \mathrm{~B}$ and $\mathrm{C}$ ). The remaining peaks at larger angles, which came mainly from the internal structures of the nucleosomes, were unchanged (Figure 3C). Indeed, a ribosome fraction from Xenopus egg extracts produced a sharp peak around $30 \mathrm{~nm}$ (Figure 3D). Our results clearly indicated that the $30-\mathrm{nm}$ peak in SAXS was derived from aggregates of ribosomes and not from the chromosomes themselves (Figure 3E). Therefore, we concluded that almost no $30-\mathrm{nm}$ chromatin structures exist in mitotic chromosomes.

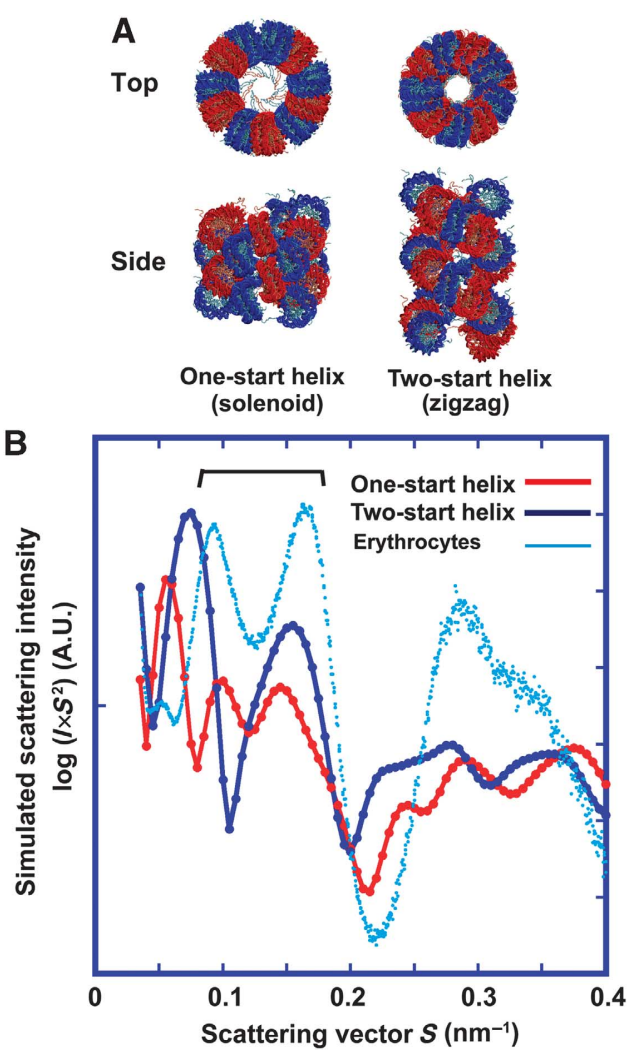

Figure 6 Computer simulation of two 30-nm chromatin fibre models. (A) Two well-known structural models of 30-nm chromatin fibres: the one-start helix (solenoid, left) and two-start helix (zigzag ribbon, right). Their atomic coordinate models (kindly provided by Dr D Rhodes, LMB, UK) are shown (Schalch et al, 2005; Robinson and Rhodes, 2006). The drawings were generated using MOLSCRIPT (Kraulis, 1991). (B) Based on the atomic model, the scattering profiles obtained with the one-start helix (red line) and two-start helix (blue line) were computationally simulated (for details, see Materials and methods). As the scattering was obtained from a single 30-nm chromatin fibre model shown in (A), the 30-nm peak was not shown. Two peaks of $\sim 11$ and $\sim 6 \mathrm{~nm}$ were prominent (brackets) in the two-start model (blue line) and the chicken erythrocyte nuclei (cyan line). Note that the simulated scattering profile of the two-start helix (blue line) seemed to be qualitatively similar to that of chicken erythrocyte nuclei (cyan line).

\section{A fractal nature of mitotic chromosomes revealed by USAXS}

Although to date, no 30-nm chromatin structure has been detected, the question remains whether any larger regular structures exist in the mitotic chromosomes. To address the challenge of investigating an entire chromosomal region by X-ray scattering, we developed a new instrument for USAXS at SPring-8. Figure 4A shows a schematic representation of the experimental setup. We examined the performance of this instrument using $\sim 1000-\mathrm{nm}$ latex particles as a control, which produced a pattern of fringes in the range of $\sim 50-$ $1000 \mathrm{~nm}$ (Figure 4B). These fringes and their slopes were almost identical to those of the computationally simulated scattering pattern (Figure 4C), verifying that the entire chromosome diameter could be covered with this instrument. By USAXS measurement, we did not detect notable regular structures in mitotic chromosomes in the range of $\sim 50-$ $1000 \mathrm{~nm}$ (Figure 4D; Supplementary Figure S5A). Importantly, we found that the scattering pattern of chromosomes followed the power law relationship between scattering 


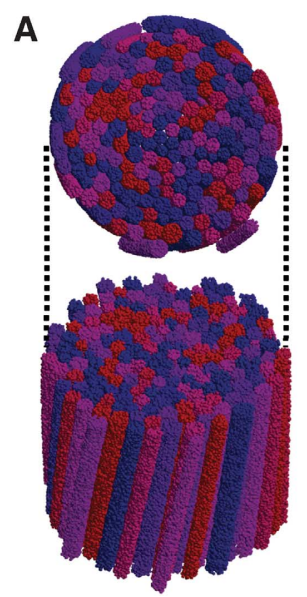

B

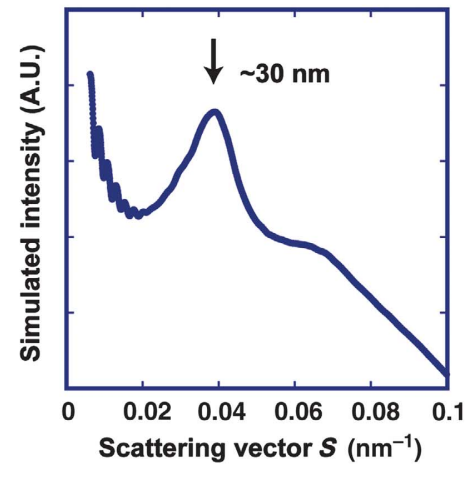

Figure 7 Computer simulation of 'loose' 30-nm fibre bundles with size and torsional variations. (A) Compact bundles of 30-nm fibres, with variations in their diameter $( \pm 15 \%)$ and torsion $( \pm 15 \%)$, were computationally modelled (for details, see Materials and methods). The upper image is the top view, and the bottom is the side view. (B) The modelled 30-nm fibre bundles produced a peak at $\sim 30 \mathrm{~nm}$ (for details, see Materials and methods).

intensity and scattering vector (the inverse of structure size) over a very wide range (Figure 4E; Supplementary Figure S5B) (Schmidt, 1989). This scattering property suggested irregular folding of nucleosome fibres, with a fractal nature.

\section{Discussion}

In the present study, to complement the structural information obtained by cryo-EM, we exploited the highly penetrative power of X-rays to explore the bulk structure of mitotic chromosomes in solution over a wide range (from nanometres to micrometres). Combining our cryo-EM, SAXS and USAXS results yielded 6 - and $11-\mathrm{nm}$ peaks but none $>11 \mathrm{~nm}$, demonstrating that chromosomes do not have notable regular structures on a chromosome-diameter scale. We suggest that the organization of mitotic chromosomes is irregular arrangements of the nucleosome (beads on a string) fibre, with a fractal nature (Figure 5A). This notion may be compatible with two recently proposed fractal organization models of interphase nuclei (Bancaud et al, 2009; Lieberman-Aiden et al, 2009; see also Takahashi, 1989). Although these three models came from different aspects (different procedures and cell-cycle phases) and their direct comparison is difficult, we consider that the evidence obtained from the different angles would strengthen the fractal concept.

In the control study, we observed a 30-nm chromatin structure in chicken erythrocyte nuclei by SAXS and cryoEM. The erythrocyte nuclei did not contain detectable ribosomes (Supplementary Figure S6). In the 30-nm chromatin fibre, intra-fibre nucleosomal association was dominant (Figure 5B). Considering the structural model in Figure 6A (Schalch et al, 2005; Robinson and Rhodes, 2006), formation of the 30-nm chromatin fibre requires that the frequencies of face-to-face ( $\sim 6$-nm peak) and edge-to-edge ( $\sim 11$-nm peak) positioning should be similar (Table I). Here, note that edgeto-edge spacing likely corresponds to the helical pitch in the 30-nm chromatin fibre. Consistent with this notion, SAXS analysis of erythrocyte nuclei showed two prominent peaks of $\sim 11$ and $\sim 6 \mathrm{~nm}$ (Figure 1A; Table I). This profile seemed to be similar to the simulated scattering profile based on the two-start helix 30-nm chromatin model (Schalch et al, 2005) (Figure 6B; Table I). In agreement with a recent cryo-EM study (Scheffer et al, 2011), this finding suggested that the nuclear organization of chromatin in chicken erythrocytes could be explained by a two-start helix 30-nm chromatin fibre. Formation of 30-nm chromatin fibres in these silenced nuclei may be a rare case involving histone modifications and/or specific protein binding for robust silencing (Thomas, 1984; Grigoryev and Woodcock, 1993; Kizilyaprak et al, 2011; Kowalski and Palyga, 2011).

In compact native chromosomes, inter-fibre nucleosome associations become increasingly dominant (Figure 5B) (Eltsov et al, 2008; Maeshima et al, 2010a). Nucleosome fibres are highly interdigitated, such that they are prevented from forming 30-nm chromatin fibres, leading to irregular folding of the nucleosome fibres (polymer melt-like structure) (Figure 5A and B) (Eltsov et al, 2008; Maeshima et al, 2010a). The scattering profile of mitotic chromosomes shows that the $\sim 6$-nm peak (face-to-face positioning) predominates over the $\sim 11$-nm peak (edge-to-edge positioning) (Figure 3C, upper; Table I). As formation of the $30-\mathrm{nm}$ chromatin fibre requires similar frequencies of face-to-face ( $\sim 6$-nm peak) and edgeto-edge $(\sim 11-\mathrm{nm}$ peak) positioning (Table I), Figure $3 \mathrm{C}$ (upper) again supports the near absence of regular $30-\mathrm{nm}$ chromatin fibres from chromosomes.

For our structural study, we assumed highly 'regular' 30-nm chromatin fibres. However, natively assembled chromatin is likely to have defects: for example, missing nucleosomes, variations in linker length and variations in posttranslational modifications. Although we cannot exclude the possibility that 'loose' 30-nm chromatin fibres may exist in mitotic chromosomes, we consider it unlikely, at least in the majority of chromosomes, because our computer simulation demonstrated that bundles of loose 30 -nm fibres with variations in their diameters $( \pm 15 \%)$ and torsions $( \pm 15 \%)$ can still produce a sharp 30-nm peak by scattering (Figure 7; for details see Materials and methods). In addition, Diesinger and Heermann (2009) reported that defects, such as missing nucleosomes, can easily disrupt 30-nm chromatin fibres.

We suggest that the organization of mitotic chromosomes involves irregular arrangements of nucleosome fibres (Figure 5A). One possible explanation why the nucleosome fibre is organized globally into a 'rod shape' (e.g., mitotic chromosome) rather than a 'sphere' is as follows. As condensin, which is essential for chromosome condensation (Hirano, 2006), forms an axis in the chromosome (Laemmli et al, 1978; Maeshima and Laemmli, 2003; Swedlow and Hirano, 2003; Ono et al, 2004; Hirota et al, 2004), it holds the nucleosome fibres globally around the chromosomal centre. If condensins bind to specific sites on the genome, forming loops that are able to interact with one another, then selfassembled axial structures will be formed (Laemmli et al, 1978). Alternatively, the formation of regular loops could be sufficient to generate a rod shape, as the loops would repel one another due to entropic effects (Marko and Siggia, 1997; Kleckner et al, 2004). The chromosome would then acquire a reproducible rod shape. The loops are collapsed and irregularly folded to make a domain (red line in Figure 5A) that may be compatible with the large module observed by the Belmont group (Strukov et al, 2003). 
Irregular folding predicts an increase in the dynamics and flexibility of the nucleosome fibre (Eltsov et al, 2008; Maeshima et al, 2010a), as small objects such as nucleosomes have fewer physical constraints than larger regular structures, such as $30-n m$ chromatin fibres. These properties of the nucleosome fibre could act as a physical driving force in chromosome assembly. We and also Fussner et al (2011) have postulated that a similar irregular folding state exists in the majority of active interphase nuclei (Maeshima et al, 2010a). In contrast to the regular folding of classical models, irregular folding implies a high degree of DNA accessibility and may have several advantages in template-directed biological processes in interphase nuclei, including RNA transcription and DNA replication, repair and recombination. For example, in transcriptional regulation, the dynamic movement of nucleosome fibres will help with the targeting of transcription complexes because target sequences are more often exposed, while regular folding structures such as $30-\mathrm{nm}$ chromatin fibres can hide target sequences. Dynamic irregular folding can also easily form loops, prompting promoter and enhancer interactions.

\section{Materials and methods}

HeLa-chromosome preparation and immunofluorescence study Mitotic chromosomes were isolated from HeLa cells essentially as described previously (Langmore and Paulson, 1983; Maeshima and Laemmli, 2003; Maeshima et al, 2005). To avoid the effects of prolonged mitotic arrest with nocodazole, a brief $(2 \mathrm{~h})$ treatment with the drug followed by mitotic shake-off was also used to collect mitotic HeLa cells. To resolve the long-standing discrepancies between cryo-EM and SAXS, the SAXS protocol of Langmore and Paulson (1983) was reproduced exactly (Paulson and Langmore, 1983). Isolated chromosomes were placed in IB (10 mM HEPES$\mathrm{KOH}$ (pH 7.5), $10 \mathrm{mM} \mathrm{NaCl}, 5 \mathrm{mM} \mathrm{MgCl} 2,0.1 \mathrm{mM}$ phenylmethylsulphonyl fluoride (PMSF) and $0.1 \%$ NP40) and subjected to SAXS measurements. Isolated chromosomes maintained under more physiological conditions (10 mM HEPES-KOH (pH 7.5), $100 \mathrm{mM}$ $\mathrm{NaCl}, 5 \mathrm{mM} \mathrm{MgCl} 2,0.1 \mathrm{mM}$ PMSF and $0.1 \%$ NP40) produced a similar scattering profile with the same three peaks (Supplementary Figure S2A and B). To remove ribosome aggregates from chromosome surfaces, isolated chromosomes were suspended briefly in buffer A (15 mM Tris-HCl (pH 7.5), $80 \mathrm{mM} \mathrm{KCl,} 2 \mathrm{mM}$ EDTA, $2 \mathrm{mM}$ spermine, $5 \mathrm{mM}$ spermidine, $0.1 \mathrm{mM}$ PMSF and $0.05 \%$ digitonin), then centrifuged and resuspended in IB for measurement. Langmore and Paulson (1983) tested several conditions (Paulson and Langmore, 1983). We were able to consistently reproduce their SAXS profiles (e.g., Supplementary Figure S2A and B; other conditions are not shown). Chicken erythrocyte nuclei were prepared and measured as described by Langmore and Schutt (1980). Ribosome fractions were isolated from Xenopus egg extracts by ultracentrifugation (kindly provided by Dr T Hirano, RIKEN).

For immunofluorescence, isolated chromosomes were suspended in HMK buffer (10 mM HEPES-KOH (pH 7.4), 1 mM $\mathrm{MgCl}_{2}$ and $100 \mathrm{mM} \mathrm{KCl}$ ), attached to poly-L-lysine-coated coverslips by centrifugation at $400 \mathrm{~g}$ for $5 \mathrm{~min}$, and fixed in HMK buffer including $1 \%$ formaldehyde. Immunofluorescence was performed as described by Maeshima et al (2006, 2010c). Image stacks were recorded with a DeltaVision microscope (Applied Precision) using a step size of $0.2 \mu \mathrm{m}$ with $\mathrm{a} \times 100 / 1.40$ UPlanSApo objective (Olympus) and deconvolved.

\section{SAXS and USAXS experiments}

SAXS and USAXS experiments were performed at SPring-8 using the BL45XU and BL29XUL beamlines, respectively. Following the approach of Langmore and Paulson (1983) (Paulson and Langmore, 1983), most of the SAXS and USAXS data in this paper are shown as plots of $\log \left(I \times S^{2}\right)$ versus $S$, obtained after subtracting buffer scattering. Here, $I$ and $S$ are the average intensity and size of the scattering vector, respectively. $I \times S^{2}$ gives the true relative strength (power) of the structural periodicities in the samples. An arbitrary periodicity of $D \mathrm{~nm}$ in the object gives rise to a peak in the X-ray scattering at a reciprocal distance $(1 / D) \mathrm{nm}^{-1}$. Thus, ' $30-\mathrm{nm}$ peak' refers to a scattering peak at $S=0.033 \mathrm{~nm}^{-1}$. The data were averaged within concentric annuli of different radii about the experimental centre to yield the average intensity $I$ as a function of $S$.

BL45XU was set up for the SAXS experiment as follows (Fujisawa et al, 2000). The X-ray wavelength and sample-to-detector distances were $0.9 \AA(13.8 \mathrm{keV})$ and $2.1 \mathrm{~m}$ (Figures $1 \mathrm{~A}$ and $\mathrm{E}$ and $3 \mathrm{C}$ and $\mathrm{D}$ ) or $3.6 \mathrm{~m}$ (Figure 1F; Supplementary Figure S2A and B), respectively. HeLa-chromosome samples were collected in quartz glass capillaries ( $2 \mathrm{~mm}$ in diameter; Tohso) by gentle centrifugation, and the chromosome regions were exposed to the X-ray beam for $1 \mathrm{~s}$. Scattering data for the chromosome samples and buffer were collected at room temperature using an imaging plate system (R-AXIS IV ++; Rigaku) (Figures $1 \mathrm{~A}$ and $\mathrm{E}$ and $3 \mathrm{C}$ and $\mathrm{D}$ ) or a cooled charge-coupled device (CCD) equipped with an X-ray image intensifier (Fujisawa et al, 2000) (Figure 1F; Supplementary Figure S2A and B).

For USAXS measurements, a coherent X-ray diffraction instrument (Nishino et al, 2009a, b) was used for high-quality data acquisition in the ultrasmall-angle region. Figure 4A shows the experimental setup. A monochromatic $8 \mathrm{keV}$ X-ray beam illuminated the HeLa-chromosome pellet through a $20-\mu \mathrm{m}$ pinhole. As described above, the chromosome pellets were formed in quartz glass capillaries ( $1 \mathrm{~mm}$ in diameter; Tohso), which were then sealed with epoxy resin (Araldite; KONISHI). The chromosome regions in the capillary were exposed to the X-ray beam for $10 \mathrm{~s}$ to obtain small-angle data, and for $100 \mathrm{~s}$ to obtain large-angle data. Small-angle scattering from the sample was recorded with a directillumination CCD detector $1.95 \mathrm{~m}$ downstream of the sample. To remove parasitic scattering from the pinhole, the instrument was equipped with two L-shaped guard slits and a beamstop to block the quadrant area of the CCD detector. The entire instrument, including the chromosome samples, was maintained in a vacuum to avoid air scattering noise.

\section{Cryo-EM and image analysis}

Chromosome freezing and cryosectioning were performed essentially as described previously (Eltsov et al, 2008). Chromosome suspensions were transferred into gold-plated copper carriers with a $0.1-\mathrm{mm}$ indentation (Engineering Office $\mathrm{M}$ Wohlwend $\mathrm{GmbH}$ ) and cryo-fixed using a HPM010 high-pressure freezer (Boeckeler Instruments). Vitreous sections were collected on C-flat grids (Protochips) or Lacey Carbon Grids (Polysciences) using a charging device (Haug Static Control Products). Grids with the cryosections were mounted on a Gatan 626 cryoholder (Gatan), and the samples were observed using a CM 100 transmission electron microscope (FEI). Vitreous sections with minimal cutting distortion were selected for image acquisition. Images of the chromosomes were recorded at $100 \mathrm{kV}$ with a $2 \mathrm{k} \times 2 \mathrm{k}$ TemCam-F224HD CCD camera (Tietz Video and Image Processing Systems). The contrast transfer function was corrected, and one-dimensional rotationally averaged power spectra of the images were calculated as described previously (Eltsov et al, 2008). The contrast transfer function was corrected by deconvolution using CTFMIX. Power spectra of individual images were calculated using SumPS (Conway and Steven, 1999) and averaged using KaleidaGraph 4.0 (Synergy Software).

\section{Computer simulation of latex particles and model scattering} profiles

The USAXS scattering pattern of latex particles was simulated using the following equation:

$$
I=\frac{16 \pi^{2}(\sin (\pi S L)-\pi S L \cos (\pi S L))^{2}}{(\pi S L)^{6}}
$$

where $L$ is the diameter of a single latex particle. The distribution of the diameter was assumed to be Gaussian, with mean and standard deviation of 1010 and $10 \mathrm{~nm}$, respectively (Figure 4C).

SAXS profiles of the interdigitated one-start helix model and two-start helical crossed-linker model were examined using a computer simulation based on their atomic coordinates (gifts from Dr Rhodes, LMB, UK). SAXS profiles of the two models were calculated with the following equation:

$$
I=\sum_{i, j} f_{i}(S) f_{j}(S) \frac{\sin \left(2 \pi S r_{i j}\right)}{2 \pi S r_{i j}},
$$

where $f_{i}(S)$ and $r_{i j}$ are the form factor of the $i$ th atom and the distance between the $i$ th and $j$ th atoms, respectively. Here, the summation was over non-hydrogen atoms in the model (Figure 6B). 


\section{Computer simulation of an $\sim 30-n m$ fibre bundle and model scattering profiles}

To confirm that the $\sim 30-\mathrm{nm}$ fibre bundles produced the $\sim 30-\mathrm{nm}$ peak, a computer simulation was performed (Figure $7 \mathrm{~B}$ ). In the simulation, finer structures (i.e., those below $\sim 30$-nm resolution) were not considered. The $\sim 30-\mathrm{nm}$ fibre bundle was modelled as follows. (i) A set of two-dimensional circles with a random Gaussian distribution of diameters with a peak $\sim 30 \mathrm{~nm}$ was generated. (ii) The spatial distribution of the circles was optimized using the simulatedannealing molecular dynamics (MD) simulation in two dimensions. (iii) From the set of circles, a bundle of fibres was generated in three dimensions. In this process, the height of the bundle was randomly determined, and all fibres were tilted slightly around the origin. The distributions of the height and tilt angles were Gaussian. (iv) Numerous pseudo-particles were generated randomly and uniformly inside each fibre at a density of $\sim 0.01$ particles $\mathrm{nm}^{-3}$. The table below summarizes the modelling parameters. The spatial distribution of the pseudo-particles modelled above was then used to compute the SAXS pattern, $I$, with the following equation:

$$
I=\exp \left(-4 \pi^{2} \sigma^{2} S^{2}\right) \sum_{i, j} \frac{\sin \left(2 \pi S r_{i j}\right)}{2 \pi S r_{i j}},
$$

where $r_{i j}$ is the distance between the $i$-th and $j$-th particles. Here, it was assumed that the electron density distribution of a pseudoparticle is Gaussian around the centre, with a standard deviation $(\sigma)$ of $3 \mathrm{~nm}$. SAXS profiles for the 1000 bundles of $\sim 30$-nm fibres were simulated and averaged.

Parameters used in computer simulations:

\begin{tabular}{lcc}
\hline & Mean value & s.d. \\
\hline Fibre diameter & $30 \mathrm{~nm}$ & $4.5 \mathrm{~nm}$ \\
Number of fibres & 200 & 14 \\
Bundle height & $400 \mathrm{~nm}$ & $60 \mathrm{~nm}$ \\
Tilt angle around the origin & $15^{\circ}$ & $2.25^{\circ}$ \\
\hline
\end{tabular}

\section{References}

Alberts B, Johnson A, Lewis J, Raff M, Roberts K, Walter P (2007) Molecular Biology of the Cell, Fifth Edition. New York: Garland Science

Bancaud A, Huet S, Daigle N, Mozziconacci J, Beaudouin J, Ellenberg J (2009) Molecular crowding affects diffusion and binding of nuclear proteins in heterochromatin and reveals the fractal organization of chromatin. EMBO J 28: 3785-3798

Bordas J, Perez-Grau L, Koch MH, Vega MC, Nave C (1986) The superstructure of chromatin and its condensation mechanism. II. Theoretical analysis of the X-ray scattering patterns and model calculations. Eur Biophys J 13: 175-185

Conway JF, Steven AC (1999) Methods for reconstructing density maps of 'single' particles from cryoelectron micrographs to subnanometer resolution. J Struct Biol 128: 106-118

Davey CA, Sargent DF, Luger K, Maeder AW, Richmond TJ (2002) Solvent mediated interactions in the structure of the nucleosome core particle at $1.9 \AA$ resolution. $J \mathrm{Mol}$ Biol 319: 1097-1113

Diesinger PM, Heermann DW (2009) Depletion effects massively change chromatin properties and influence genome folding. Biophys J 97: 2146-2153

Dubochet J, Sartori Blanc N (2001) The cell in absence of aggregation artifacts. Micron 32: 91-99

Eltsov M, Maclellan KM, Maeshima K, Frangakis AS, Dubochet J (2008) Analysis of cryo-electron microscopy images does not support the existence of $30-\mathrm{nm}$ chromatin fibers in mitotic chromosomes in situ. Proc Natl Acad Sci USA 105: 19732-19737

Finch JT, Klug A (1976) Solenoidal model for superstructure in chromatin. Proc Natl Acad Sci USA 73: 1897-1901

Fujisawa $\mathrm{T}$, Inoue $\mathrm{K}$, Oka $\mathrm{T}$, Iwamoto $\mathrm{H}$, Uruga $\mathrm{T}$, Kumasaka $\mathrm{T}$, Inoko Y, Yagi N, Yamamoto M, Ueki T (2000) Small-angle X-ray scattering station at the SPring-8 RIKEN beamline. $J$ Appl Cryst 33: $797-800$

Fussner E, Ching RW, Bazett-Jones DP (2011) Living without $30 \mathrm{~nm}$ chromatin fibers. Trends Biochem Sci 36: 1-6
Supplementary data

Supplementary data are available at The EMBO Journal Online (http://www.embojournal.org).

\section{Acknowledgements}

We are grateful to $\mathrm{T}$ Fujisawa, $\mathrm{T}$ Hayakawa, $\mathrm{J}$ Dubochet for technical support and K Yokomizo, A Watanabe and Y Sasaki for providing technical assistance. We would like to thank D Rhodes for providing models of the 30-nm fibres; $\mathrm{T}$ Uchiumi for the anti-P antibody; T Hirano for providing the Xenopus ribosome fraction; $\mathrm{B}$ Earnshaw for DT40 cells; S Jun, B Stern, J Kim, T Cremer, T Sutani and $M$ Shimura for critical reading of the manuscript; and $Y$ Hiraoka, J Cooper, K Nasmyth, A Belmont, N Kleckner, J Paulson, $M$ Yamamoto and M Nakasako for helpful discussion and support. We also thank I Hihara for his professional illustrations. This work was supported by a grant-in-aid for the Promotion of XFEL Research of MEXT, a MEXT grant, CREST and a MHLW grant for Advanced Medical Technology. ME and ASF were supported by the 3D-EM Network of Excellence within Research Framework Program 6 of the European Commission. HT and SH are JSPS fellow.

Author contributions: KM designed the project. KI and KM performed the SAXS measurements at BL45 of SPring-8. ME performed the cryo-EM study and analysed the image data. YN developed the apparatus for USAXS. YN, YT, KI and KM performed the USAXS measurements at BL29 of SPring-8. YJ performed the computer simulation. YJ, KI and KM analysed the scattering data. HT, KM and SH performed the immunostaining experiment. YN and ME provided essential suggestions throughout the project. ASF, NI and TI provided advice and support on the project. KM wrote the manuscript.

\section{Conflict of interest}

The authors declare that they have no conflict of interest.

Gilbert N, Boyle S, Fiegler H, Woodfine K, Carter NP, Bickmore WA (2004) Chromatin architecture of the human genome: gene-rich domains are enriched in open chromatin fibers. Cell 118: $555-566$

Grigoryev SA, Woodcock CL (1993) Stage-specific expression and localization of MENT, a nuclear protein associated with chromatin condensation in terminally differentiating avian erythroid cells. Exp Cell Res 206: 335-343

Hirano T (2006) At the heart of the chromosome: SMC proteins in action. Nat Rev Mol Cell Biol 7: 311-322

Hirota T, Gerlich D, Koch B, Ellenberg J, Peters JM (2004) Distinct functions of condensin I and II in mitotic chromosome assembly. J Cell Sci 117: 6435-6445

Kireeva N, Lakonishok M, Kireev I, Hirano T, Belmont AS (2004) Visualization of early chromosome condensation: a hierarchical folding, axial glue model of chromosome structure. J Cell Biol 166: $775-785$

Kizilyaprak C, Spehner D, Devys D, Schultz P (2011) In vivo chromatin organization of mouse rod photoreceptors correlates with histone modifications. PLoS One 5: e11039

Kleckner N, Zickler D, Jones GH, Dekker J, Padmore R, Henle J, Hutchinson J (2004) A mechanical basis for chromosome function. Proc Natl Acad Sci USA 101: 12592-12597

Kornberg RD, Lorch Y (1999) Twenty-five years of the nucleosome, fundamental particle of the eukaryote chromosome. Cell 98: 285-294

Kowalski A, Palyga J (2011) Chromatin compaction in terminally differentiated avian blood cells: the role of linker histone $\mathrm{H} 5$ and non-histone protein MENT. Chromosome Res 19: 579-590

Kraulis PJ (1991) MOLSCRIPT: a program to produce both detailed and schematic plots of protein structures. J Appl Cryst 24: $946-950$

Laemmli UK, Cheng SM, Adolph KW, Paulson JR, Brown JA, Baumbach WR (1978) Metaphase chromosome structure: the 
role of nonhistone proteins. Cold Spring Harb Symp Quant Biol 42: $351-360$

Langmore JP, Paulson JR (1983) Low angle x-ray diffraction studies of chromatin structure in vivo and in isolated nuclei and metaphase chromosomes. J Cell Biol 96: 1120-1131

Langmore JP, Schutt C (1980) The higher order structure of chicken erythrocyte chromosomes in vivo. Nature 288: 620-622

Lewis CD, Laemmli UK (1982) Higher order metaphase chromosome structure: evidence for metalloprotein interactions. Cell 29: 171-181

Lieberman-Aiden E, van Berkum NL, Williams L, Imakaev M, Ragoczy T, Telling A, Amit I, Lajoie BR, Sabo PJ, Dorschner MO, Sandstrom R, Bernstein B, Bender MA, Groudine M, Gnirke A, Stamatoyannopoulos J, Mirny LA, Lander ES, Dekker J (2009) Comprehensive mapping of long-range interactions reveals folding principles of the human genome. Science 326: 289-293

Maeshima K, Eltsov M (2008) Packaging the genome: the structure of mitotic chromosomes. J Biochem (Tokyo) 143: 145-153

Maeshima K, Laemmli UK (2003) A two-step scaffolding model for mitotic chromosome assembly. Dev Cell 4: 467-480

Maeshima K, Eltsov M, Laemmli UK (2005) Chromosome structure: improved immunolabeling for electron microscopy. Chromosoma 114: $365-375$

Maeshima K, Hihara S, Eltsov M (2010a) Chromatin structure: does the 30-nm fibre exist in vivo? Curr Opin Cell Biol 22: 291-297

Maeshima K, Hihara S, Takata H (2010b) New insight into the mitotic chromosome structure: irregular folding of nucleosome fibers without 30-nm chromatin fiber. Cold Spring Harb Symp Quant Biol 75: 439-444

Maeshima $\mathrm{K}$, Iino $\mathrm{H}$, Hihara $\mathrm{S}$, Funakoshi $\mathrm{T}$, Watanabe $\mathrm{A}$, Nishimura M, Nakatomi R, Yahata K, Imamoto F, Hashikawa T, Yokota H, Imamoto N (2010c) Nuclear pore formation but not nuclear growth is governed by cyclin-dependent kinases (Cdks) during interphase. Nat Struct Mol Biol 17: 1065-1071

Maeshima K, Yahata K, Sasaki Y, Nakatomi R, Tachibana T, Hashikawa T, Imamoto F, Imamoto $\mathrm{N}$ (2006) Cell-cycledependent dynamics of nuclear pores: pore-free islands and lamins. J Cell Sci 119: 4442-4451

Marko JF, Siggia ED (1997) Polymer models of meiotic and mitotic chromosomes. Mol Biol Cell 8: 2217-2231

McDowall AW, Smith JM, Dubochet J (1986) Cryo-electron microscopy of vitrified chromosomes in situ. EMBO J 5: 1395-1402

Nishino Y, Takahashi Y, Imamoto N, Ishikawa T, Maeshima K (2009a) Three-dimensional visualization of a human chromosome using coherent X-ray diffraction. Phys Rev Lett 102: 18101

Nishino Y, Takahashi Y, Kubo H, Furukawa H, Yamauchi K, Maeshima K, Imamoto N, Hirohata R, Matsubara E, Ishikawa T (2009b) Nanostructure analysis by coherent hard X-ray diffraction. J Phys 186: 012056
Ohta S, Wood L, Bukowski-Wills JC, Rappsilber J, Earnshaw WC (2010) Building mitotic chromosomes. Curr Opin Cell Biol 23: $114-121$

Ono T, Fang Y, Spector DL, Hirano T (2004) Spatial and temporal regulation of condensins I and II in mitotic chromosome assembly in human cells. Mol Biol Cell 15: 3296-3308

Paulson JR, Langmore JP (1983) Low angle x-ray diffraction studies of HeLa metaphase chromosomes: effects of histone phosphorylation and chromosome isolation procedure. J Cell Biol 96: 1132-1137

Robinson PJ, Rhodes D (2006) Structure of the ' $30 \mathrm{~nm}$ ' chromatin fibre: a key role for the linker histone. Curr Opin Struct Biol 16: 336-343

Robinson PJ, Fairall L, Huynh VA, Rhodes D (2006) EM measurements define the dimensions of the ' $30-\mathrm{nm}$ ' chromatin fiber: evidence for a compact, interdigitated structure. Proc Natl Acad Sci USA 103: 6506-6511

Schalch T, Duda S, Sargent DF, Richmond TJ (2005) X-ray structure of a tetranucleosome and its implications for the chromatin fibre. Nature 436: 138-141

Scheffer MP, Eltsov M, Frangakis AS (2011) Evidence for short-range helical order in the 30-nm chromatin fibers of erythrocyte nuclei. Proc Natl Acad Sci USA 108: 16992-16997

Schmidt PW (1989) Use of scattering to determine the fractal dimension. In The Fractal Approach to Heterogeneous Chemistry, Avnir D (ed), pp 67-79. Chichester: John Wiley \& Sons

Strukov YG, Wang Y, Belmont AS (2003) Engineered chromosome regions with altered sequence composition demonstrate hierarchical large-scale folding within metaphase chromosomes. J Cell Biol 162: 23-35

Swedlow JR, Hirano T (2003) The making of the mitotic chromosome: modern insights into classical questions. Mol Cell 11: $557-569$

Takahashi M (1989) A fractal model of chromosomes and chromosomal DNA replication. $J$ Theor Biol 141: 117-136

Thomas JO (1984) The higher order structure of chromatin and histone H1. J Cell Sci Suppl 1: 1-20

Tremethick DJ (2007) Higher-order structures of chromatin: the elusive $30 \mathrm{~nm}$ fiber. Cell 128: 651-654

Uchiumi T, Traut RR, Kominami R (1990) Monoclonal antibodies against acidic phosphoproteins P0, P1, and P2 of eukaryotic ribosomes as functional probes. J Biol Chem 265: 89-95

Widom J, Klug A (1985) Structure of the 300A chromatin filament: X-ray diffraction from oriented samples. Cell 43: 207-213

Woodcock CL (1994) Chromatin fibers observed in situ in frozen hydrated sections. Native fiber diameter is not correlated with nucleosome repeat length. J Cell Biol 125: 11-19

Woodcock CL, Frado LL, Rattner JB (1984) The higher-order structure of chromatin: evidence for a helical ribbon arrangement. J Cell Biol 99: 42-52 